\title{
Hard Exudate Extraction from Fundus Images using Watershed Transform
}

\author{
Vasanthi Satyananda ${ }^{1}$, K V Narayanaswamy ${ }^{2}$, Karibasappa ${ }^{3}$ \\ ${ }^{1}$ Associate Professor, Atria Institute of Technology, Bangalore, Karnataka, India \\ ${ }^{2}$ Principal, Atria Institute of Technology, Bangalore, Karnataka, India \\ ${ }^{3}$ Dayanand Sagar University, Bangalore, Karnataka, India
}

\begin{tabular}{l} 
Article Info \\
\hline Article history: \\
Received Oct 22, 2018 \\
Revised Apr 30, 2019 \\
Accepted Sept 5, 2019 \\
\hline Keyword: \\
Exudates \\
Watershed \\
Extended Maxima \\
Fundus \\
Diabetic Retinopathy \\
FPGA
\end{tabular}

Corresponding Author:

Vasanthi Satyananda,

Associate Professor,

Atria Institute of Technology,

Bangalore, Karnataka, India,

Email: vasanti67@gmail.com

\begin{abstract}
Diabetic Retinopathy is a medical condition which affects the eyes due to increased blood sugar levels. This is characterized by presence of exudates deposits of lipids in the posterior pole of the retina. If this ailment is not treated in earlier stages these deposits can cause blurred vision or even permanent blindness. This paper concentrates on extraction of hard exudates and optic disc from the retinal images of eyes using Marker based Watershed approach, which uses the minima imposition method to create mask and marker. The varying contrast across all the images has been taken care by a non-linear equation. Once these bright objects have been extracted from fundus images, area estimation is performed to eliminate the optic disk, thus retaining only exudates. These images have been procured from publicly available databases. Though software systems are easy to install, they prove to be expensive in terms of time and cost; thus this method has also been implemented on FPGA for an on-chip solution. The precision and sensitivity for exudate extraction sans optic disk are found to be $92.4 \%$ and $83.78 \%$ respectively. Though other techniques exist which provide better accuracy, the method described in this paper is found to be hardware friendly in comparison with other proven methods. Few steps of the algorithm developed are implemented on FPGA to provide an embedded system approach to this work, considering the advantages of a hardware-software combination.
\end{abstract}

Copyright $\odot 2018$ Institute of Advanced Engineering and Science. All rights reserved.

\section{INTRODUCTION}

Commonly known as "Diabetes", Diabetes mellitus is a medical malady caused due to non-typical levels of blood glucose level [1]. The main reason to be listed for this ailment is lack of insulin secretion by the pancreatic gland, which helps the body monitor the glucose level in blood. Diabetes affects several parts of the body such as kidneys, eyes and nerves.

Diabetic Retinopathy (abbreviated as DR) is one such medical abnormality where the blood vessels of the retina of the eyes become fragile and eventually disintegrate thus causing swelling or leakage of the blood vessel fibre ending [2]. This also terminates the oxygen flow to retina thus causing the death of retinal tissues. The accumulation of leaked fats and lipids is known as exudates. There are two types of DR: Non Proliferative DR (NPDR) and Proliferative DR (PDR) [3]. NPDR is an early stage of DR where the retina inflames due to leakage of blood vessels, especially in the macular region of the eye, known as macular edema [4]. Also, the blood vessels can bloat and eventually leading to closure of the same, which is named as macular ischemia [5]. This leads to deposits of fatty compounds and lipids in the retina, which are known as exudates. NPDR often results in blurry vision. PDR is the progressive stage of DR. This is characterised by an intricate 
condition known as neovascularization [6]. Here, new fragile blood vessels grow in the retinal area. Excessive bleeding caused by these vessels can result in permanent blindness. Furthermore, these vessels can combine to become scar tissue, which eventually detach the retina.

There are two types of exudates: Hard exudates and soft exudates [7]. Also known as waxy exudates, hard exudates are shiny deposits found in the posterior pole of the eye. These bright white or yellowish white deposits with sharp margins often have roundish patterns. Their size is diverse - found either as small or huge patches. On the other hand, soft exudates, often harmless, are often caused due to ischemia. These grayish white deposits have irregular, cloud like shapes. This characteristic fetches these exudates the name "Cotton Wool Spots".

The images of retina or the back of the eyes are captured using a fundus camera [8]. The severity of exudates is measured based on its area [9]. If the total area of the exudates is less than the quarter area of the optic disc, then it is diagnosed as mild spread. If the exudate area ranges between quarter and a full disc area, then it is diagnosed to be moderate. There shall be reason for alarm when the exudate spread area is more than the area of the optic disc.

Since the evolution of digital image processing, its contribution towards medical imaging and diagnosis is immense. The increasing growth rate of DR and exudates amongst individuals and an intention of successful mass screening of exudates led to the invasion of image processing into fundus image analysis for the first time in the year 1997 [10]. Since then, this computer assisted diagnosis technique has explored several algorithms to extract exudates from the retinal area. The main idea of this paper is to extract both exudates and optic disc, ignoring other complications found in the fundus images.

As mentioned earlier, several algorithms and techniques have been or are being proposed to extract high contrast artefacts that represent exudates and optic disc. Soman and Ravi [11] propose that optic disc can be extracted from the green channel component of the original colour image by utilizing Hough transform [12] and bit plane slicing. Exudates can be extracted using multiple thresholds. The resulting binary image can be smoothened using morphological opening and Gaussian filter. According to Vimala and Mohideen [13], median filtering is applied on the intensity component of HIS colour model to obtain uniformly illuminated image using a 3x3 window. Contrast limited adaptive histogram equalization (CLAHE) [14] is performed on the resultant of median filter This component is merged with hue and saturation and the combination is converted to $\mathrm{L}^{*} \mathrm{a}^{*} \mathrm{~b}^{*}$ colour space. Different objects from $\mathrm{a}^{*} \mathrm{~b}^{*}$ layers have to be segmented using K-means clustering method [15]. The pixels of these clusters are labelled with an index obtained after k-means clustering. Based on homogeneous colour property, exudates and optic disc are extracted. Furthermore, Support Vector Machine (SVM) is employed to detect exudates using features such as energy, dissimilarity, homogeneity and standard deviation.

Esmaeili, et. al., [16] suggest the application of digital curvelet transform (DCUT) [17] for better extraction of high intensity pixels from an image. Initially, Haar transform [17] is applied on the red plane with empirical threshold (value $=35$ ). Morphological operations are executed such that this process results in a binary image. Fourier samples of the RGB image are obtained by performing two-dimensional Fast Fourier Transform (2D FFT). When digital curvelet transform (DCUT) [18] is applied on this contrast enhanced green image, coefficients with greater value when compared to the rest are obtained in the areas of optic disc and exudates due to their high contrast. Based on the coefficients, the image is reconstructed and the RGB colour model is configured back. Next, CLAHE is applied to enhance optic disc (OD) further from red and green channels. Canny edge detector and morphological operators are applied to extract optic disc. The OD is localised by locating the converging points of all the blood vessels in the retina. In [19], Mohamed et. al. propose to resize the image to reduce computation time. CLAHE is applied over green channel to enhance contrast. White top hat transform with huge structuring element is applied to avoid loss of information, if huge exudates are found to be present. However, small exudates lost during this process are obtained by performing logical ORing of binary image obtained with morphological gradient applied on green channel.

Elbalaoui, et al., in [20] recommend that red and green channels for OD extraction. Both features are combined using a mathematical relation. The OD is localised using Curvature Scale Space (CSS) [21]. 2D Vesselness function as described in [22] is used to eliminate blood vessels with which the pixels of blood vessels are replaced with neighbouring non-blood vessel pixels. Later, Local Binary Fitting [23] to obtain active contours of OD is applied. A similar approach has been showcased in [24]. In [25], histogram matching method is opted for OD detection. The image is denoised using a mean filter. After its conversion to grayscale, OD area is manually cropped. Histograms of these cropped areas are computed are stored as templates. As part of testing, several images are divided into 80x80 squares and histograms are plotted. These histograms are compared with templates previously stored. The area with best match is considered to be OD. The found area is eliminated in the RGB image by replacing the OD pixels with black pixels. Green channel is extracted from edited RGB image and CLAHE is applied to improve the contrast of the image. The image is divided into 2 halves vertically and histogram is computed for both halves. The difference between these peaks is estimated 
and the peak obtained is fed to Otsu's algorithm [26] to get binary image. A similar approach has been proposed in [27] where HIS colour model has been used instead of grayscale.

According to [28], the original image is converted to the HSV colour model and median filtering is applied. On this image, a brightness correction equation is applied. Further, the original image is normalised and summed up with a brightness controlled image. Gamma correction is applied to neutralise brightness fluctuations. The green channel of the image is extracted and segmentation is performed to extract optic disk and exudates. The threshold for this process is estimated using histogram. To eliminate optic disk, the red component is considered and CLAHE is applied, followed by median filtering. Thresholding is performed using histogram analysis and the blob with largest area is removed. This resulting image is used as mask to extract only exudates from the previously segmented image.

As per paper [29], the green channel is extracted from the fundus image and average filtering is performed to remove Gaussian noise in the image. Next, every pixel of the image is compared with a certain 'threshold'; if the pixel value is above this threshold, then the pixel is multiplied with an integer factor, else it is divided by the same integer factor. As exudates are of higher pixel value, these blobs get highlighted even more, while the background gets suppressed into darker areas. Segmentation is performed on this image to extract exudates. Morphological operations such as erosion and dilation are performed to nullify small and unnecessary white blobs segmented. Also the optic disk, which is often disintegrated into fragments due to blood vessels flowing through it, are brought back together using binary dilation and hole filling. This image is subjected to edge detection. The blob with longest perimeter is declared optic disk and is eliminated. Furthermore, various approaches have been described in the earlier work, as a survey paper [30].

The papers mentioned above purely concentrate on software approaches for extracting exudates. It can be clearly seen that predicting and classifying algorithms have been utilised to obtain results. These solutions are usually performed using general-purpose processors (GPPs). The basic advantage of using GPPs is that any of the parameters of the developed procedures can be modified anytime without much effort. Though these methods may provide accurate outcomes, they cannot be employed if one has to implement a hardware approach, preferably on an FPGA, to enhance the working speeds [31]. Conversely, the huge size of images and the mathematical complexity involved in these algorithms do not permit the designers or the developers to build a hundred percent hardware solutions. Thus, considering the advantages of both hardware and software, a feasible embedded system solution is proposed in this paper. Watershed based approach is applied to extract the bright lesions from fundus images. As these lesions involve both exudates and optic disc, area based optic disc elimination is performed to eliminate optic disc and retain only the exudates. Sections 2 and 3 focus on proposed methodology, and FPGA implementation respectively. Section 4 highlights the results of the work conducted. Section 5 concludes the paper.

\section{PROPOSED METHODOLOGY}

\subsection{Image Acquisition}

The fundus images have been procured from publicly available databases -DiaRetDB0 [32], DiaRetDB1 [33], IDRiD [34] and MESSIDOR [35]. The original image consists of true colors - red, green and blue, each with 8 bit values, which makes a pixel's bitwidth 24. As processing 24 bits per pixel can be heavy on the processor, a colour layer of 8 bit per pixel in which exudates stand out from the background has to be chosen [36].

\subsection{Colour Component Selection}

RGB, YCbCr and HSV colour models are considered here. YCbCr, also recognised as ITU-R BT.601, consists of $\mathrm{Y}$ component, known as Luma component, represents the brightness present in the colour. $\mathrm{Cb}$ and $\mathrm{Cr}$ are the chroma components; $\mathrm{Cb}$ is chroma blue which is the difference between blueness of the colour and the brightness, while $\mathrm{Cr}$ (chroma red) is the redness minus brightness. The RGB colour image is converted to YCbCr using the following equations [37].

$$
\begin{aligned}
& Y=0.299 R+0.587 G+0.114 B \\
& C b=0.16 R-0.33 G+0.5 B \\
& C r=0.5 R-0.418688 G-0.081312 B
\end{aligned}
$$

Hue, Saturation and Intensity (HIS) colour layers are closely related to human's perception of colours. Hue represents different colours. Saturation is the purity of the colours. Intensity, also called value (V) represents brightness of the colour [38]. The RGB image is converted to HIS model using the following equations:

$$
H=\left\{\begin{array}{cl}
\theta, & \text { if } B \leq G \\
360-\theta, & \text { otherwise }
\end{array}\right.
$$


where,

$$
\begin{aligned}
& \theta=\cos ^{-1} \frac{0.5((R-G)+(R-B))}{\sqrt{(R-G)^{2}+(R-G)(G-B)}} \\
& S=1-\frac{3 * \min (R, G, B)}{R+G+B} \\
& V=\frac{R+G+B}{3}
\end{aligned}
$$

Figure 1 represents different colour aspects of a fundus image. Green channel is chosen as it is noticed for 70 images that this layer prominently highlighted OD and exudates.

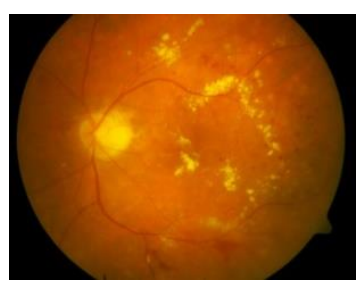

(a)

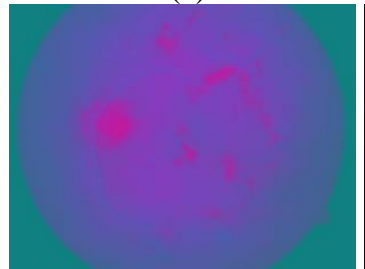

(e)

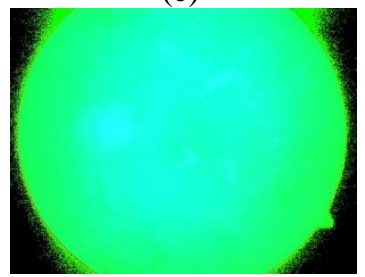

(i)

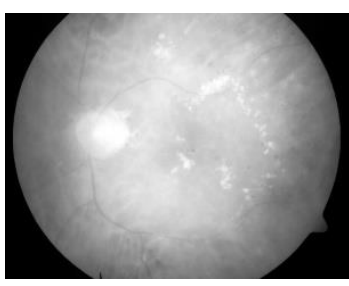

(b)

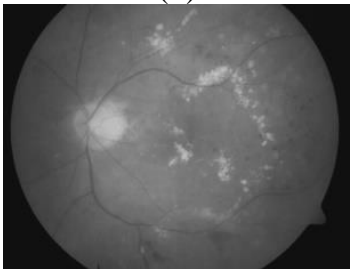

(f)

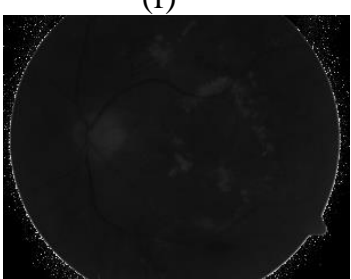

(j)

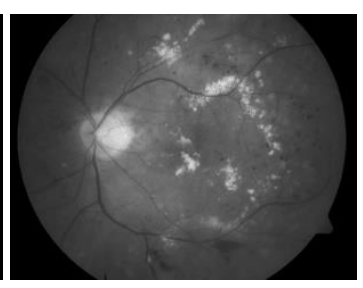

(c)

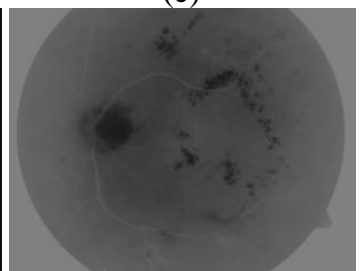

(g)

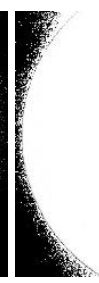

(k)

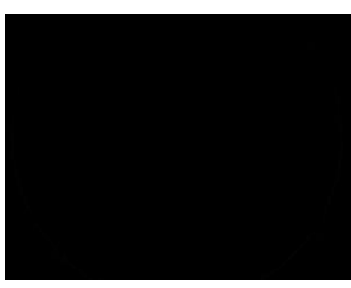

(d)

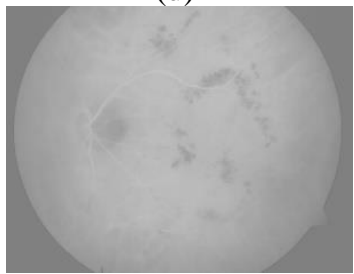

(h)

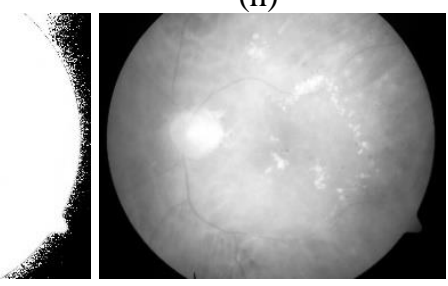

(1)

Figure 1. (a) RGB image, (b) R image, (c) G image, (d) B image, (e) YCbCr image, (f) Y image, (g) $\mathrm{Cb}$ image, (h) $\mathrm{Cr}$ image, (i) HIS image, (j) H image, (k) $\mathrm{S}$ image and (l) V image

\subsection{Removal of Blemishes in the Image}

It can be clearly noticed in Figure $1 \mathrm{~b}$ that the image, along with comprising of exudates and OD, also consists of bright blemishes. In order to remove these blotches mean filtering technique is employed. The image of size $1152 \times 1500$ is divided into small image patches of size $10 \times 10$. For each image patch, the mean of all pixels is calculated and all pixels are replaced with the average value obtained [39]. Figure 2a shows the outcome of this procedure.

\subsection{Contrast Correction}

While extracting the exudates and the optic disc, the non-uniform illumination of the background can pose a threat for accurate analysis. Thus, in order to normalise the background's contrast, contrast correction has to be performed with the help of Non-linear Brightness Transform [40], as shown in Figure 2b. If out is the output pixel, in is input pixel, $n$ is number of bits required to represent a pixel and $\alpha$ is a numerical, where $\alpha \in$ $(0,1)$, then the transform is represented as,

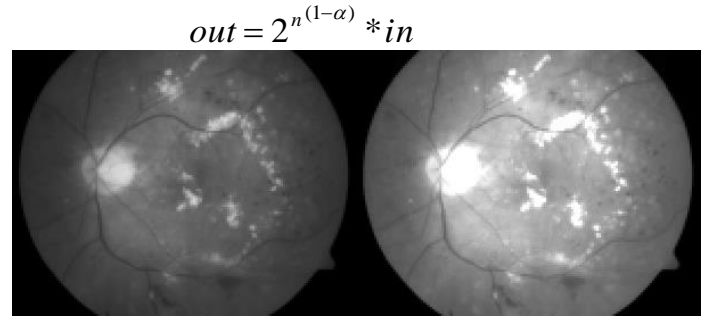

(a)

(b)

Figure 2. (a) Average filtered image (b) Contrast corrected image 


\subsection{Watershed Algorithm}

Watershed based segmentation is a technique where the whole image is divided into small nonoverlapping areas with the concept of watershed [41]. This is conceptualised by simulating water flood over a grayscale image. The pixel intensities form a terrain where the connected low intensity pixels, known as local minima, form "valleys" or slopes, while higher intensity pixels form edges of valleys or "ridges" and the lowest precipitate of pixels, where the water is assumed to be accumulating is considered as catchment basin. When the flooding increases, eventually the water in the catchment basins increases and can cause all the basins to merge due to overflow. At this point, dams are constructed to prevent this amalgamation, and these dams are known as watershed divides, or simply "watersheds". To perform watershed algorithm, marker based gradient approach has been proved to be the best to eliminate over segmentation [42]. A marker is a group of high contrast connected pixel components belonging to an image. This highlights the ridges of the grayscale image. There are two types of markers - internal marker that are connected components belonging to the object of interest, and external marker representing background's connected pixels. The former is obtained by estimating extended maxima of the image and latter is procured using distance transform.

\subsubsection{Extended Maxima}

Extended maxima transform is a gray level mathematical morphology transform that is defined as the regional maxima of the H-maxima transform [43]. Regional maxima are connected pixel components with an intensity value $\mathrm{P}$, where $\mathrm{P}$ is greater than its neighbourhood, thus resulting in a binary image. In case of $\mathrm{H}$ maxima, regional maxima are computed and all heights greater than $\mathrm{H}$ are clipped off. Extended maxima is performed on contrast adjusted image with $\mathrm{h}=30$ and the result is shown in Figure $3 \mathrm{a}$.

\subsubsection{Distance Tranform}

Distance transform is a technique where the Euclidian distance between zero and nearest non-zero element in a binary image is estimated [44]. The ones are replaced with zeros and zeros are replaced with distances calculated. To acquire binary image, segmentation and morphological closing is applied on the contrast adjusted image. The Euclidian distance is defined as:

$$
d=\sqrt{\left(x_{1}-x_{2}\right)^{2}+\left(y_{1}-y_{2}\right)^{2}}
$$

\subsubsection{Segmentation}

Since the objects of interest appear the brightest, manual thresholding can be applied to segment the exudates and optic disc. A threshold $(\mathrm{t})$ of 240 is set to make sure that all bright objects are extracted, as represented in equation (10) [45]. Figure $3 b$ shows segmented image.

$$
\text { seg }= \begin{cases}\text { white, } & \text { if } \text { pixel }_{\text {in }} \geq \mathrm{t}=240 \\ \text { black, } & \text { otherwise }\end{cases}
$$

\subsubsection{Morphological Operations}

To remove small, unwanted pixels that are neither part of OD or exudates, morphological operation has to be performed. The basic morphological operators are erosion and dilation [46]. In case of erosion, if any of the neighbouring pixels of the structuring element [47] is black, then the centre pixel is made black. In case of absence of black in the neighbourhood, the centre pixel is retained as it is. If E is eroded image, B is binary image and $\mathrm{S}$ is the structuring element, erosion is represented as follows.

$$
E=B \Theta S
$$

Dilation is the complement of erosion; the centre pixel is changed to white if at least one white pixel is found in its neighbourhood. If D is dilated image, B is binary image and $\mathrm{S}$ is the structuring element, then dilation is represented as follows.

$$
D=B \oplus S
$$

The process of performing dilation, followed by erosion on an image with a common structuring element is known as Morphological Closing. In the algorithm, closing is performed on the binary image obtained after thresholding with a circular structuring element of radius 5, as shown in Figure 3c. Distance transform is applied on the "complemented" closed image, as this operation requires the background to be white and the object to be black, as shown in Figure 3d. Watershed transform is applied on the distance transform output to obtain the external marker, as seen in Figure 3e. The next step would be to combine internal and external markers and perform minima imposition as a baseline to perform watershed based segmentation.

\subsubsection{Minima Imposition}

Minima imposition modifies the grayscale image such that regional minima occur only in marked locations [48]. All other pixel values are elevated in its intensity, hence eliminating futile regional maxima. This involves a mask and a marker. A mask is a binary image whose foreground marks the region where regional minima has to be calculated. In the case of this algorithm, the mask is the result of logical OR of the internal and external markers. The complemented contrast adjusted image is considered as the marker. Minima imposition, as seen in Figure 4f, is represented using the following equation. 


$$
o p=\text { impose }_{\min }\left(\sim \operatorname{conop},\left(e m \mid w s_{\text {dist }}\right)\right)
$$

where impose $_{\min }$ is minima imposition function, con $_{o p}$ is contrast adjusted image, em is extended maxima image and $w s_{\text {dist }}$ is watershed applied on distance transform.

\subsubsection{Watershed Transform on Minima Imposition}

Watershed transform is applied on the image resulting from minima imposition, as shown in Figure $3 \mathrm{~g}$. The watershed "divides", i.e., the borders of the overlapping blobs obtained by applying watershed, are superimposed over the closed image to separate overlapping exudates and optic disc, if any, as seen in Figure $3 \mathrm{~h}$.

In the superimposed image, the largest white blob is regarded as optic disc and is eliminated. To calculate the biggest blob, connected-component labelling [49] is carried out. As the brightest and largest area of retina is OD, the label which has repeated most number of times is estimated and this label is changed to zero. This makes all the pixels lying in the OD area black, thus resulting in extraction of hard exudates.

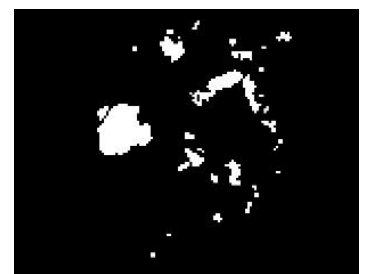

(a)

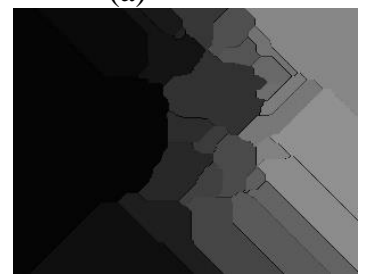

(e)

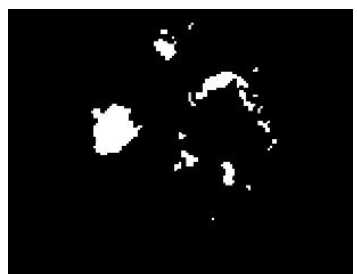

(b)

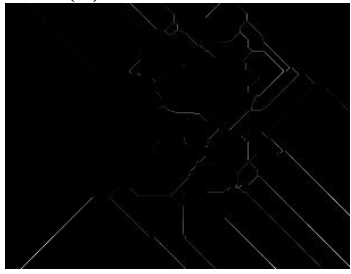

(f)

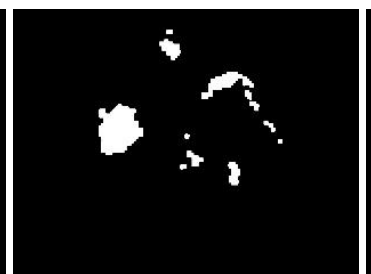

(c)

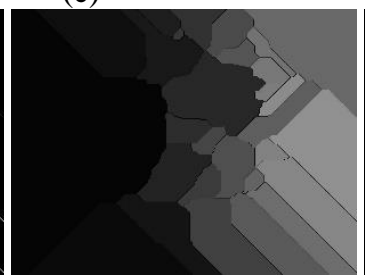

(g)

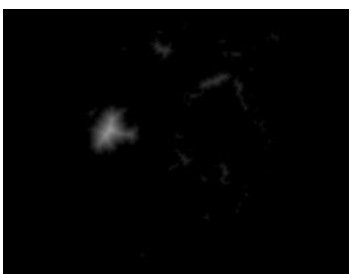

(d)

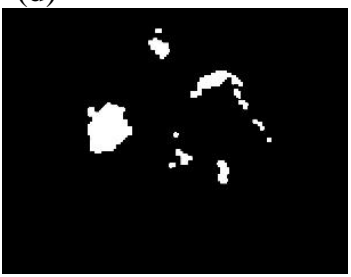

(h)

Figure 3. (a) Extended Maxima, (b) Segmentation, (c) Morphological closing, (d) Distance transform output,

(e) Watershed on distance transform, (f) Minima imposition, (g) Watershed on minima imposition and (h) Superimposition of watershed divides on closed image

\section{IMPLEMENTATION ON FPGA}

The crucial part of designing an embedded system is to categorise the steps of the algorithm into two areas - hardware implementable and software implementable. The steps that are straightforward and with minimal memory requirement are often chosen for hardware implementation. On the other hand, complex steps are preferred for software implementation. Figure 4 showcases the steps chosen for the hardware and software modules. Average filtering, contrast adjustment, segmentation, morphological closing and image complement are opted for FPGA implementation, and the remaining modules are demonstrated in software using MATLAB [50].

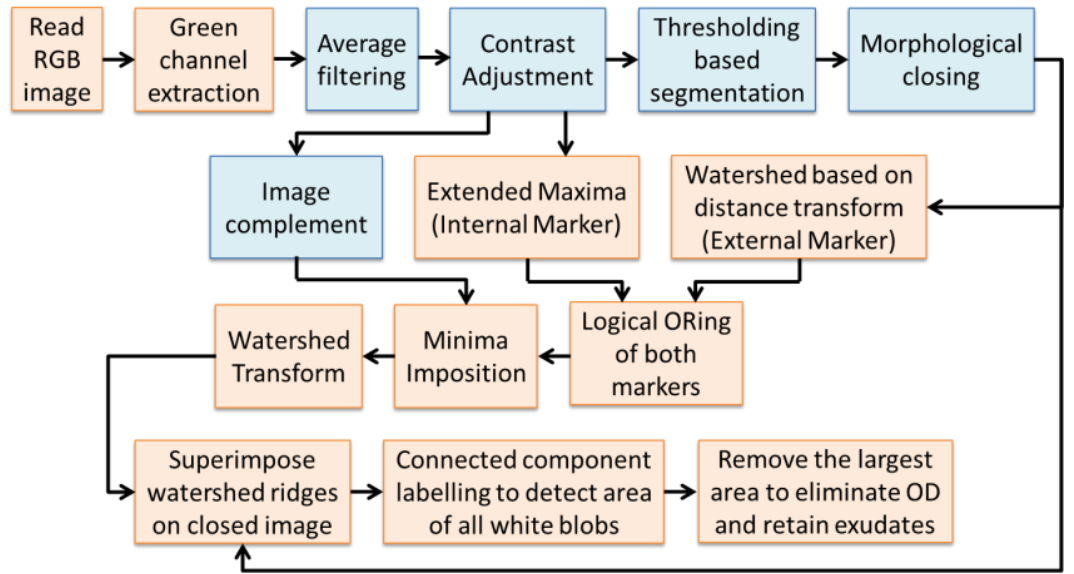

Figure 4. Differentiating the steps implemented on hardware and software; blue blocks represent FPGA development and orange blocks represent MATLAB implementation 
The green channel is extracted from the RGB image and the pixels have to be passed to the FPGA for further processing. These pixel values are stored in a block RAM (BRAM) of the FPGA through a coefficient file.

\subsection{Average Filtering}

For average filtering, the window size chosen is $10 \times 10$ pixels, which intends to add 100 pixel values and divide the sum by 100. The flowchart for this operation is shown in Figure 5, as per which 99 adder units are utilised for addition and division is performed using the restoring method [51].

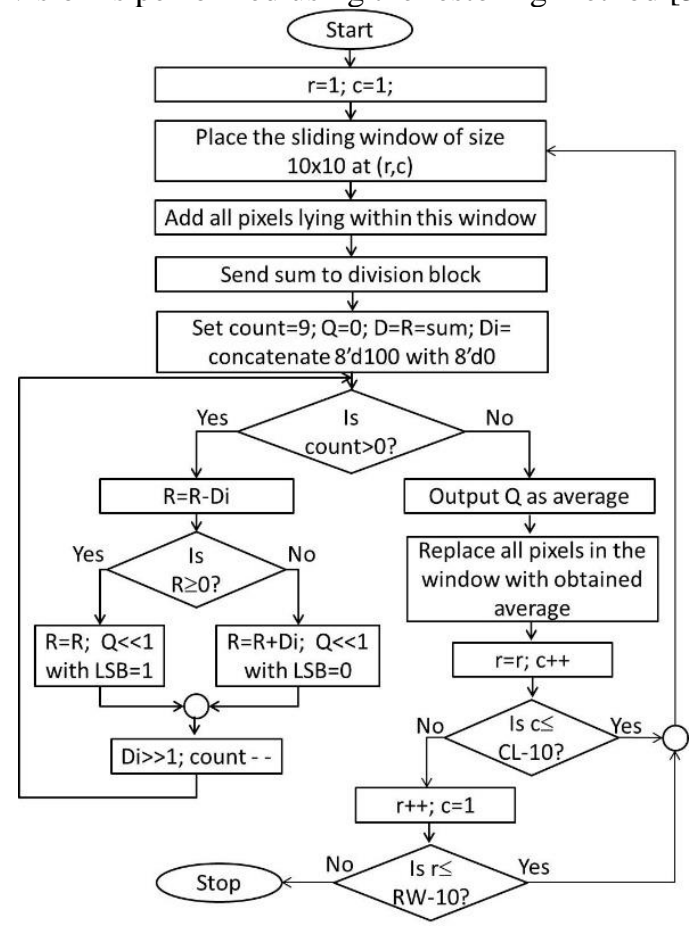

Figure 5. Flowchart depicting average filtering using restoring division, where $r$ is the row number of the pixel in consideration, $\mathrm{c}$ is the column number of the pixel, RW and CL are total number of rows and columns respectively, Q, D, Di and R indicate quotient, dividend, divisior and remainder respectively.

\subsection{Contrast Adjustment}

For the grayscale image, $n$ is 8 bits in equation (8), and $\alpha$ chosen is 0.9 . Substituting these values, the constant approximately equates to 1.75 , which can represented as $1+0.5+0.25$. These coefficients can be represented in powers of two as $2^{0}+2^{-1}+2^{-2}$. Powers of two are converted to shifting operations as shown in equation (14). Shifters and adders are utilised for this operation, as shown in Figure 6.

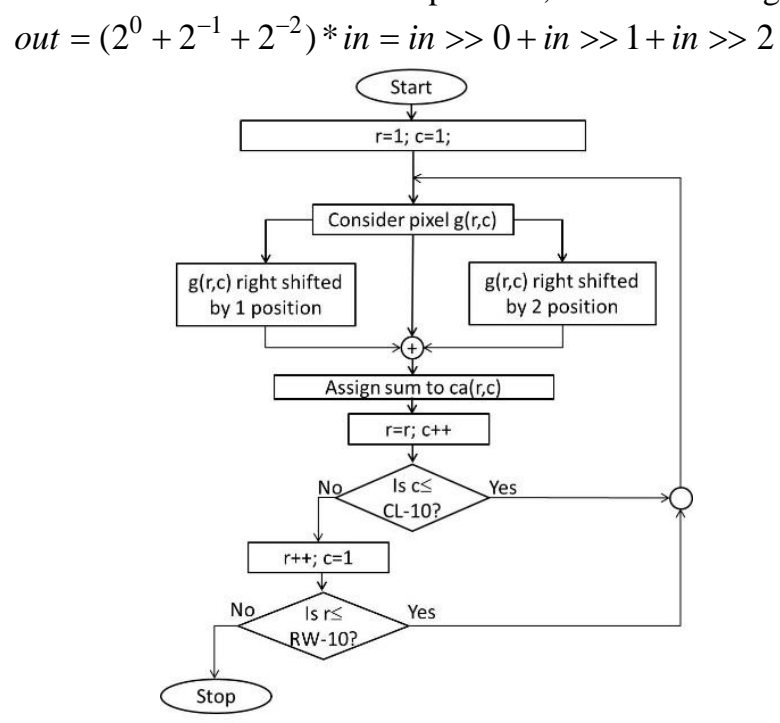


Figure 6. Flowchart showing the working of contrast adjustment, where $g(r, c)$ is a pixel of green component

\subsection{Thresholding} located at $\mathrm{r}^{\text {th }}$ row and $\mathrm{c}^{\text {th }}$ column and ca is the contrast adjusted image

Here, each pixel is compared with a threshold. If the pixel is greater than or equal to threshold selected, then the resultant pixel with same coordinates is assigned a ' 1 ', indicating a white pixel. On the other hand, the pixel with lesser intensity value than the threshold is assigned a ' 0 ' indicating a black pixel. This operation is shown in Figure 7, where $\mathrm{ca}$ and seg are the contrast adjusted image and segmented image respectively.

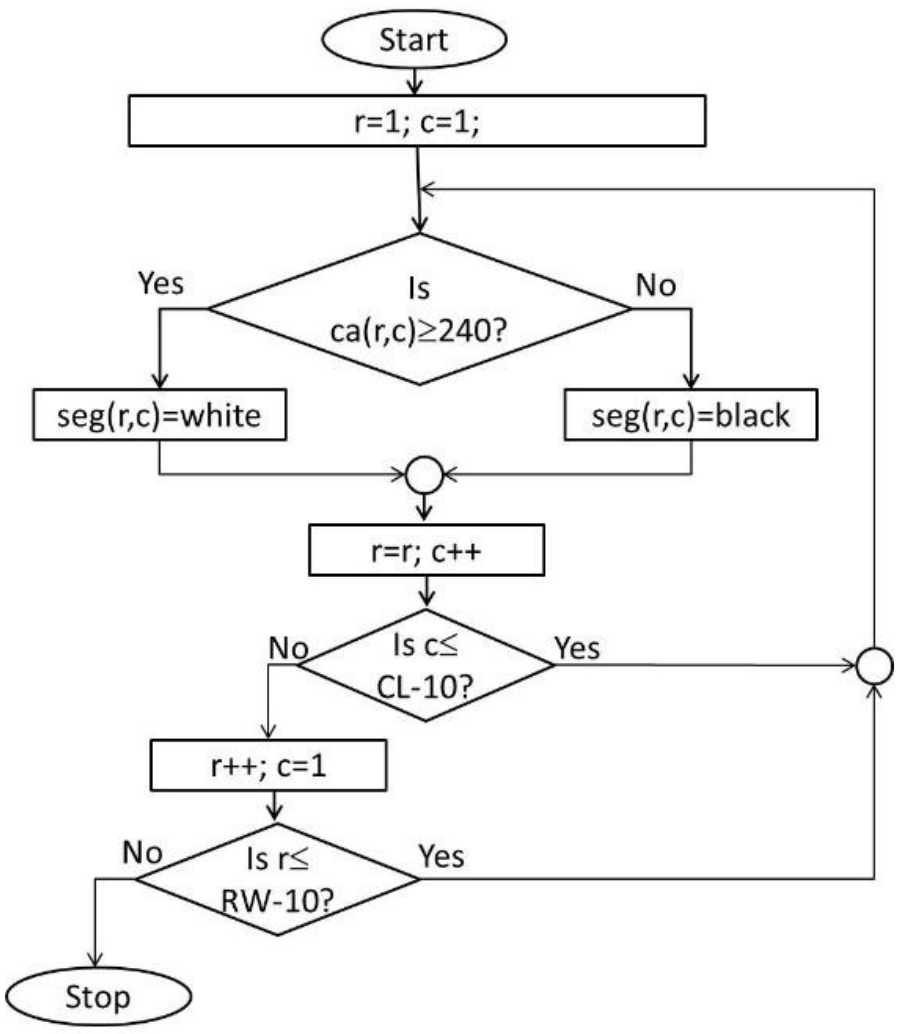

Figure 7. Flowchart depicting segmentation, with threshold set as 240.

\subsection{Morphological Closing}

As mentioned in the previous section, morphological operation is the operation of performing erosion on the dilated image. A common disk shaped structuring element of radius 5 is selected for this operation, which appears like the matrix shown in Figure 8.

\begin{tabular}{|l|l|l|l|l|l|l|l|l|}
\hline 0 & 0 & 1 & 1 & 1 & 1 & 1 & 0 & 0 \\
\hline 0 & 1 & 1 & 1 & 1 & 1 & 1 & 1 & 0 \\
\hline 1 & 1 & 1 & 1 & 1 & 1 & 1 & 1 & 1 \\
\hline 1 & 1 & 1 & 1 & 1 & 1 & 1 & 1 & 1 \\
\hline 1 & 1 & 1 & 1 & 1 & 1 & 1 & 1 & 1 \\
\hline 1 & 1 & 1 & 1 & 1 & 1 & 1 & 1 & 1 \\
\hline 1 & 1 & 1 & 1 & 1 & 1 & 1 & 1 & 1 \\
\hline 0 & 1 & 1 & 1 & 1 & 1 & 1 & 1 & 0 \\
\hline 0 & 0 & 1 & 1 & 1 & 1 & 1 & 0 & 0 \\
\hline
\end{tabular}

Neighbouring

pixels

\section{Centre pixel}

Pixels not to

be considered

Figure 8. Matrix representing disk shaped Structuring element of radius 5 


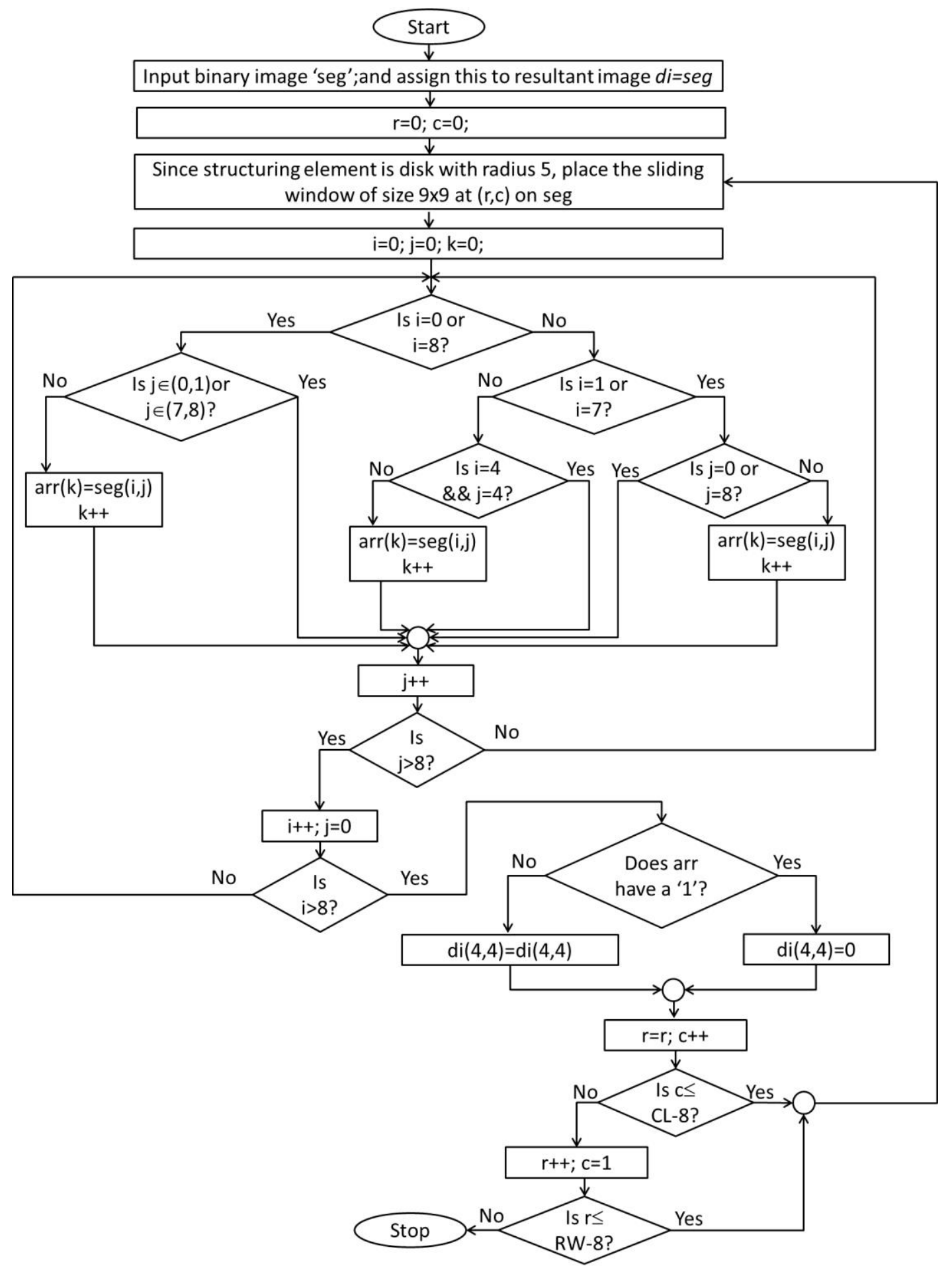

Figure 9. Flowchart depicting dilation, where seg and $d i$ are segmented image and dilated image respectively.

The neighbouring pixels that correspond to the coefficients (highlighted using orange boxes as per figure 8) of the structuring element are arranged into a one-dimensional array. In case of dilation, if the array consists of atleast one ' 1 ' value, then the centre pixel (corresponding to blue box of structuring element as per Figure 7) is assigned the value ' 1 ', as shown in Figure 9. On the contrary, in case of erosion, if the array consists of atleast one ' 0 ' value, then the centre pixel is assigned the value ' 0 ', as shown in Figure 10. 


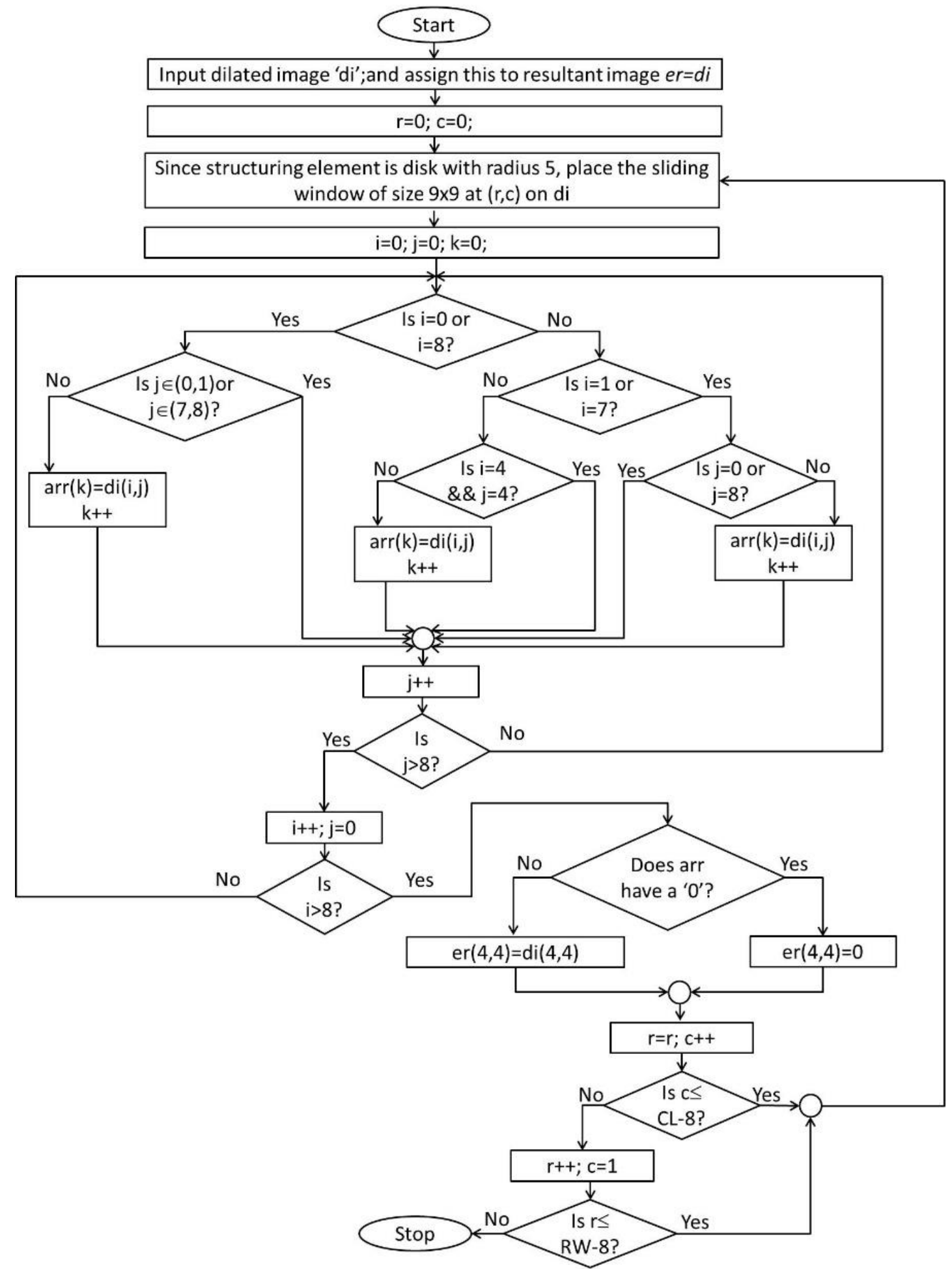

Figure 10. Flowchart depicting erosion, where $d i$ and $e r$ are dilated image and eroded image respectively. 


\subsection{Image Complement}

The complement of the image is calculated by subtracting each pixel from 255 , as summarised in Figure 11.

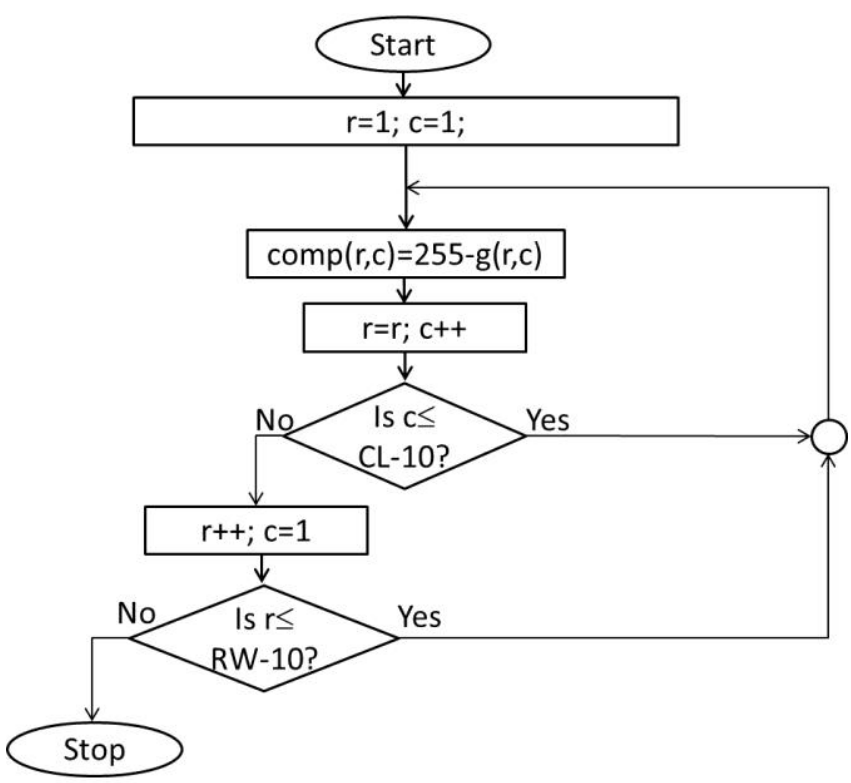

Figure 11. Image complement flowchart where, comp and $g$ are complemented image result and $g$ is green component.

After each step, the results are stored in BRAM. The pixel data obtained from the image resulting from the closing operation and the data of the complemented image are taken into MATLAB as a .dat file.

\section{RESULTS}

This algorithm has been tested for 70 images, out of which hard exudates were successfully extracted from 63 images, as shown in Figure 12a and Figure 12b. This conclusion is attained by visually comparing the areas of exudates obtained using algorithm and the areas of exudates sensed in the RGB images. Also few cases failed due to enormous fluctuations of intensity, because of which few exudates have been lost. As it can be seen in Figure 12c and Figure 12d, this method fails for images with a greenish tinge. Furthermore, if the optic disk lies in dark contrast area while the exudates lie in the brighter region of the image, the optic disk will never be identified and the largest exudate area will be wrongly considered as the optic disk and in turn will be eliminated as can be seen in Figure 12e and Figure 12f. Thus, the accuracy as per the visual analysis can be said to be $89.85 \%$. However, to further validate the results, precision and sensitivity (also known as recall) of the algorithm is also calculated. If TP, FP and FN represent true positives, false positives and false negatives respectively, precision and sensitivity are as shown in equation $(15)[52,53]$, and are found to be $92.4 \%$ and $83.78 \%$ respectively by comparing the results obtained with the ground truths provided in the database.

$$
\text { Precision }=\frac{T P}{T P+F P} \quad \text { and } \quad \text { Sensitivity }=\frac{T P}{T P+F N}
$$

Table 1 shows the time delay and area utilization of the architectures simulated on Artix 7 FPGA (XC7A100T) [54]. The timing constraint is set to be 10 nanoseconds, using Vivado 2017.2.

Table 1. Synthesis Summary

\begin{tabular}{lcc}
\hline \multicolumn{3}{c}{ Table 1. Synthesis Summary } \\
\hline Average filter & $\begin{array}{c}\text { Time Delay } \\
\text { (nanoseconds) }\end{array}$ & Area (\%) \\
Contrast Adjustment & 8.802 & 1.440 \\
Thresholding & 2.031 & 0.010 \\
Erosion & 2.192 & 0.003 \\
Dilation & 3.857 & 0.123 \\
Image Complement & 3.857 & 0.123 \\
\hline
\end{tabular}




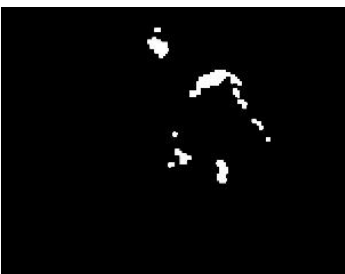

(a)

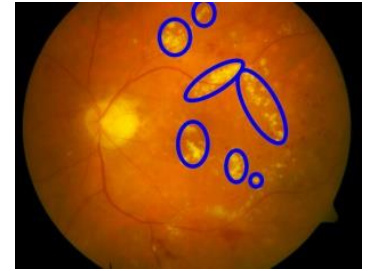

(b)

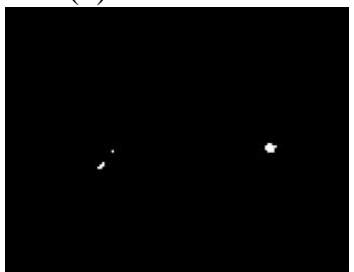

(e)

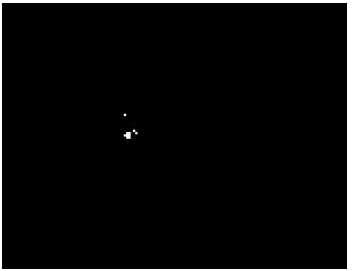

(c)

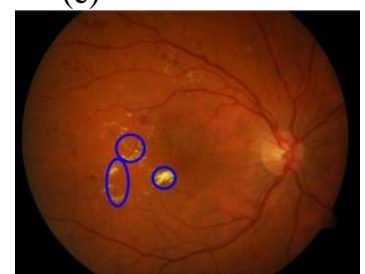

(f)

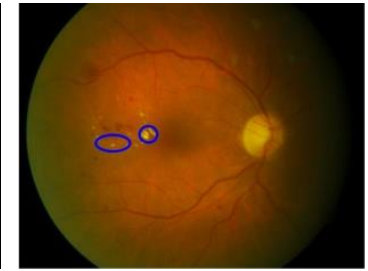

(d)

Figure 12. (a,c,e) Exudates extracted using algorithm and (b,d,f) Area of exudates marked for visual verification.

\section{CONCLUSION}

Based on the results, it can be concluded that the algorithm works well when the brightness is sufficiently uniform and the colour of the fundus images is either in bright shades of yellow or orange. It is also noticed that the soft exudates are eliminated by the averaging filter to smoothen the area. Though smaller window size can be used for mean filter, it is highly undesirable to do so as the blemishes of image remain unattended. Thus, there arises a need to explore other pre-processing techniques to highlight OD and exudates irrespective of the brightness or the contrast of original images.

The fact that the precision obtained from this algorithm is low in comparison with the methodologies established earlier by other researchers is absolutely true; this algorithm has been developed with an intention to create a hardware circuitry for analysis of exudates in fundus images. The major purpose of implementing the steps of this algorithm on a FPGA is to make sure that the diagnosis cost and speed are made more efficient rather than that of the software approaches researches have been dealing with for almost over two decades.

\section{REFERENCES}

[1] Shouip, Hossam, Diabetes mellitus, 2014.

[2] K. Boyd, "What is Diabetic Retinopathy", American Academy of Ophthalmology, Sept 01, 2017. Available Online: https://www.aao.org/eye-health /diseases/what-is-diabetic-retinopathy

[3] L. Aiello, T. W. Gardner, G. L King, G. Blankenship, J. Cavallerano, F. Ferris, and R. Klein, "Diabetic Retinopathy" Diabetes care, 21. 143-56. 10.2337/diacare.26.2007.S99, 1998.

[4] D. J. Browning, Diabetic Macular Edema. In: Browning D. (eds) Diabetic Retinopathy. Springer, New York, NY, 2010.

[5] P. Romero-Aroca, M. Baget-Bernaldiz, A. Pareja-Rios, M. Lopez-Galvez, R. Navarro-Gil, and R. Verges, "Diabetic Macular Edema Pathophysiology: Vasogenic versus Inflammatory," Journal of Diabetes Research, vol. 2016, Article ID 2156273, 17 pages, 2016.

[6] A. Ishibazawa, et al. "Characteristics of Retinal Neovascularization in Proliferative Diabetic Retinopathy Imaged by Optical Coherence Tomography Angiography.” Investigative ophthalmology \& visual science 5714 (2016): 62476255.

[7] V. Esmann, K. Lundbaek, P. H. Madsen, “Types of exudates in diabetic retinopathy,” Acta Med Scand. 1963;174:37584.

[8] N. Panwar, P. Huang, J. Lee, P. Keane, T. Swee Chuan, A. Richhariya, S. Teoh, T. Han Lim, and R. Agrawal, "Fundus Photography in the 21 st Century-A Review of Recent Technological Advances and Their Implications for Worldwide Healthcare," Telemedicine journal and e-health : the official journal of the American Telemedicine Association, 22. 10.1089/tmj.2015.0068, 2015.

[9] "The COMS Grading Scheme: Graded Features", Carver College of Medicine, The University of Iowa, 2006. Available Online: http://webeye.ophth.uiowa.edu/ dept/coms/grading/hard-exudates.htm

[10] C. Zheng Liu, Opas and S. M. Krishnan, "Automatic image analysis of fundus photograph," Engineering in Medicine and Biology Society, 1997. Proceedings of the 19th Annual International Conference of the IEEE, Chicago, IL, 1997, pp. 524-525 vol.2.

[11] K. Soman and D. Ravi, "Detection of exudates in human fundus image with a comparative study on methods for the optic disk detection," International Conference on Information Communication and Embedded Systems (ICICES2014), Chennai, 2014, pp. 1-5. 
[12] J. Illingworth and J. Kittler, "The Adaptive Hough Transform," in IEEE Transactions on Pattern Analysis and Machine Intelligence, vol. PAMI-9, no. 5, pp. 700-708, Sept. 1987.

[13] G. S. A. G. Vimala and S. K. Mohideen, "Automatic detection of Optic Disk and Exudate from retinal images using Clustering algorithm," 2013 7th International Conference on Intelligent Systems and Control (ISCO), Coimbatore, Tamil Nadu, India, 2013, pp. 280-284.

[14] K. Zuiderveld, "Contrast limited adaptive histogram equalization”. In Graphics gems IV, Paul S. Heckbert (Ed.). Academic Press Professional, Inc., San Diego, CA, USA 474-485, 1994.

[15] G. A. Wilkin and X. Huang, "K-Means Clustering Algorithms: Implementation and Comparison," Second International Multi-Symposiums on Computer and Computational Sciences (IMSCCS 2007), Iowa City, IA, 2007, pp. 133-136.

[16] M. Esmaeili, H. Rabbani, A. M. Dehnavi and A. Dehghani, "Automatic detection of exudates and optic disk in retinal images using curvelet transform," in IET Image Processing, vol. 6, no. 7, pp. 1005-1013, October 2012

[17] D. Donoho \& M. R. Duncan, "Digital Curvelet Transform: Strategy, Implementation and Experiments," Proceedings of SPIE - The International Society for Optical Engineering, 2000.

[18] P. Porwik and A. Lisowska, The Haar-wavelet transform in digital image processing: its status and achievements, 13, 2004.

[19] B. Mohamed, C. Yazid, B. Nourreddine, B. Abdelmalek and C. Assia, "Non-proliferative diabetic retinopathy detection using mathematical morphology," 2018 IEEE 4th Middle East Conference on Biomedical Engineering (MECBME), Tunis, Tunisia, 2018, pp. 219-224.

[20] A. Elbalaoui, Y. Ouadid and M. Fakir, "Segmentation of Optic Disc from Fundus Images," 2018 International Conference on Computing Sciences and Engineering (ICCSE), Kuwait City, 2018, pp. 1-7.

[21] Elbalaoui, M. Fakir, M. Boutaounte, and A. Merbouha, "Automatic Localization of the Optic Disc Center in Retinal Images based on Angle Detection in Curvature Scale Space,” JECO 13(2), (2015) 1-13.

[22] Elbalaoui, M. Fakir, M. Boutaounte and A. Merbouha, "Automatic Detection of Blood Vessel in Retinal Images using vesselness enhancement filter and Adaptive thresholding," IJHISI 12(1) (2016) 2-12.

[23] C. Li, C. Y. Kao, J. C. Gore and Z. Ding, "Implicit Active Contours Driven by Local Binary Fitting Energy," 2007 IEEE Conference on Computer Vision and Pattern Recognition, Minneapolis, MN, 2007, pp. 1-7.

[24] A. Elbalaoui, M. Fakir, Y. Ouadid, K. Taifi and A. Merbouha, "Boundary Segmentation of Optic Disc in Fundus Images," 2017 14th International Conference on Computer Graphics, Imaging and Visualization, Marrakesh, 2017, pp. 62-68.

[25] K. Y. Win and S. Choomchuay, "Automated detection of exudates using histogram analysis for Digital Retinal Images," 2016 International Symposium on Intelligent Signal Processing and Communication Systems (ISPACS), Phuket, 2016, pp. 1-6.

[26] N. Otsu, "A Threshold Selection Method from Gray-Level Histograms," in IEEE Transactions on Systems, Man, and Cybernetics, vol. 9, no. 1, pp. 62-66, Jan. 1979.

[27] H. A. Nugroho, K. Z. W. Oktoeberza, I. Ardiyanto, R. L. B. Buana and M. B. Sasongko, "Automated segmentation of hard exudates based on matched filtering," 2016 International Seminar on Sensors, Instrumentation, Measurement and Metrology (ISSIMM), Malang, 2016, pp. 84-87.

[28] D. Lokuarachchi, K. Gunarathna, L. Muthumal and T. Gamage, "Automated Detection of Exudates in Retinal Images," 2019 IEEE 15th International Colloquium on Signal Processing \& Its Applications (CSPA), Penang, Malaysia, 2019, pp. 43-47.

[29] V. Satyananda, N. K V and Karibasappa, "Exudate Extraction from Fundus Images," 2019 11th International Conference on Knowledge and Smart Technology (KST), Phuket, Thailand, 2019, pp. 94-98.

[30] V. Satyananda, K. V. Narayanaswamy, K. Karibasappa, "Extraction of Exudates from the Fundus Images A Review", in International Journal of Engineering Research \& Technology, vol. 5, no. 12, pp. 133-138, Dec 2016.

[31] V. Satyananda, K. V. Narayanaswamy and Karibasappa, "An embedded system based solution for exudate extraction," 2017 International Conference on Robotics, Automation and Sciences (ICORAS), Melaka, 2017, pp. 15.

[32] T. Kauppi, V. Kalesnykiene, J.-K. Kamarainen, L. Lensu, I. Sorri, H. Uusitalo, H. Kälviäinen, and J. Pietilä, DIARETDB0:Evaluation Database and Methodology for Diabetic Retinopathy Algorithms, Technical report

[33] T. Kauppi, V. Kalesnykiene, J.-K. Kamarainen, L. Lensu, I. Sorri, A. Raninen, R. Voutilainen, H. Uusitalo, H. Kälviäinen, J. Pietilä, DIARETDB1 diabetic retinopathy database and evaluation protocol, Technical report

[34] P. Porwal, S. Pachade, R. Kamble, M. Kokare, G. Deshmukh, V. Sahasrabuddhe, and F. Meriaudeau, Indian Diabetic Retinopathy Image Dataset (IDRiD): A Database for Diabetic Retinopathy Screening Research. Data 2018, 3, 25.

[35] Decencière, et al., "the Messidor database, Image Analysis \& Stereology," Feedback on a publicly distributed database, v. 33, n. 3, p. 231-234, aug. 2014. ISSN 1854-5165.

[36] S. R. Rupanagudi, et al., "A high speed algorithm for identifying hand gestures for an ATM input system for the blind," 2015 IEEE Bombay Section Symposium (IBSS), Mumbai, 2015, pp. 1-6.

[37] S. R. Rupanagudi et al., "A novel video processing based cost effective smart trolley system for supermarkets using FPGA," 2015 International Conference on Communication, Information \& Computing Technology (ICCICT), Mumbai, 2015, pp. 1-6.

[38] C. Liu, X. Lu, S. Ji and W. Geng, "A fog level detection method based on image HSV color histogram," 2014 IEEE International Conference on Progress in Informatics and Computing, Shanghai, 2014, pp. 373-377.

[39] G. Gupta and R. Chandel, Image Filtering Algorithms and Techniques: A Review, 2003.

[40] S. Dua, R. U. Acharya and E. Y. K. Ng, "Computational Methods in Feature Detection in Optical Images" in Computational Analysis of the Human Eye with Applications, Singapore: World Scientific Publishing, 2011, pp. 42. 
[41] B. Han, "Watershed Segmentation Algorithm Based on Morphological Gradient Reconstruction," 2015 2nd International Conference on Information Science and Control Engineering, Shanghai, 2015, pp. 533-536.

[42] W. Zhang and D. Jiang, "The marker-based watershed segmentation algorithm of ore image," 2011 IEEE 3rd International Conference on Communication Software and Networks, Xi'an, 2011, pp. 472-474.

[43] Y. Qin, W. Wang, W. Liu, and N. Yuan (2013), "Extended-Maxima Transform Watershed Segmentation Algorithm for Touching Corn Kernels", Advances in Mechanical Engineering, 5, 268046.

[44] G. J. Grevera, "Distance transform algorithms and their implementation and evaluation," Deformable Models, pp 3360 (2007)

[45] S. R. Rupanagudi, et al., "A Video Processing Based Eye Gaze Recognition Algorithm for Wheelchair Control," 2019 10th International Conference on Dependable Systems, Services and Technologies (DESSERT), Leeds, United Kingdom, 2019, pp. 241-247.

[46] S. R. Rupanagudi, V. G. Bhat, K. R. V. R. Savarni, S. Bharadwaj and V. N. P. Prasuna, "A novel automatic low cost cutting machine-cum-3D printer using an image processing based control," 2015 IEEE Bombay Section Symposium (IBSS), Mumbai, 2015, pp. 1-6.

[47] R. Srisha and A. Khan, Morphological Operations for Image Processing: Understanding and its Applications, 2013.

[48] P. Soille, "Advances in the analysis of topographic features on discrete images" in International Conference on Discrete Geometry for Computer Imagery, Springer, 2002, pp. no. 175-186.

[49] L. He, X. Ren, Q. Gao, X. Zhao, B. Yao, and Y. Chao. "The Connected-Component Labeling Problem: A Review of State-of-the-Art Algorithms." Pattern Recognition, 70:25--43, 2017

[50] V. Satyananda, K. V. Narayanaswamy, and Karibasappa, "An Embedded System for Watershed Based Hard Exudate Extraction," In: Abraham A., Cherukuri A., Melin P., Gandhi N. (eds) Intelligent Systems Design and Applications. ISDA 2018 2018. Advances in Intelligent Systems and Computing, vol 940. Springer, Cham, 2020.

[51] D. Kumar, P. Saha, A. Dandapat, "Hardware implementation of methodologies of fixed point division algorithms," International Journal on Smart Sensing and Intelligent Systems. 10. 630-645. 10.21307/ijssis-2017-227, 2017.

[52] B. Janney, G. Meera, G. Uma Shankar, S. Divakaran, S. Abraham, "Detection and classification of exudates in retinal image using image processing techniques," Journal of Chemical and Pharmaceutical Sciences. 8. 541-546, 2015.

[53] Z. N. Reza, F. Nuzhat, N. Ashfat Mahsa, and Md. Ali, "Detecting Jute Plant Disease Using Image Processing and Machine Learning," 10.1109/CEEICT.2016.7873147, 2016.

[54] 7 Series FPGAs - Configurable Logic Block, UG474 (v1.8) September 27, 2016. 